\title{
Christoph Jacob Trew - Bibliothek und Sammeln in DER Gelehrtengemeinschaft DER ERSTEN HäLfTe Des 18. JAhrhunderts
}

\author{
Elisabeth Engl - Ursula Rautenberg ${ }^{1}$
}

\section{EINLeitung}

In der Frühen Neuzeit waren Bibliotheken als soziale Orte ein bedeutendes Element im Kommunikationsgefüge der Gelehrtenrepublik. Sie dienten als Orte für regelmäßige Treffen unter den Gelehrten einer Region bzw. Stadt, wie diese besonders in der Pariser Bibliothek der Brüder Dupuy gepflegt wurden. ${ }^{2}$ Darüber hinaus wurden sie zur Anlaufstelle für Reisende, die ihre Routen teilweise nach den Empfehlungen der ars apodemica anlegten und auf ihrem Weg möglichst viele berühmte Sammlungen besuchen wollten. ${ }^{3}$ Auch die annähernd vollständige medizinisch-naturkundliche Bibliothek des Nürnberger Arztes Christoph Jacob Trew von 34.000 Titeln in knapp 24.000 Bänden war als eine der größten Ärztebibliotheken ihrer Zeit im 18. Jahrhundert weithin bekannt. ${ }^{4} \mathrm{Ob}$ diese zu einem Ort regelmäßiger Treffen wurde, ist nicht bekannt. Sie zog jedoch einige Reisende an, die sich in die fünf Stammbücher Trews eintrugen. Insgesamt enthalten diese 452 Einträge,

1 Kapitel I-III Elisabeth EngL, Kapitel IV Ursula Rautenserg.

2 Vgl. Klaus Garber: Paris, die Hauptstadt des europäischen Späthumanismus, Jaques Auguste de Thou und das Cabinet Dupuy. In: Sebastian Neumeister, Conrad Wiedemann (Hg.): Res Publica Litteraria. Die Institutionen der Gelehrsamkeit in der frühen Neuzeit. Teil I. Wiesbaden 1987 (Wolfenbütteler Arbeiten zur Barockforschung Bd. 14), S. 71-92; Stefan SiEmER: Geselligkeit und Methode. Naturgeschichtliches Sammeln im 18. Jahrhundert. Mainz 2004 (Veröffentlichungen des Instituts für Europäische Geschichte Mainz Bd. 192: Abt. für Universalgeschichte).

3 Vgl. z.B. Peter Jörg Becker: Bibliotheksreisen in Deutschland im 18. Jahrhundert. In: Archiv für Geschichte des Buchwesens 21 (1980), S. 1361-1534.

4 Vgl. Bernd Lorenz: Allgemeinbildung und Fachwissen. Deutsche Ärzte und ihre Privatbibliotheken. Herzogenrath 1992 (Studien zur Medizin-, Kunst- und Literaturgeschichte Bd. 30), S. 32-49. 
die zwischen 1724 und Trews Todesjahr 1769 angefertigt wurden. Unter den internationalen Einträgern finden sich auch einige bekannte Persönlichkeiten wie die gelehrten Ärzte Anton de Haen, Albrecht von Haller, Lorenz Heister und Abraham Vater. ${ }^{5}$ Als Sehenswürdigkeit wurde Trews Büchersammlung zumindest in zwei Reiseberichten empfohlen, die allerdings erst nach Trews Ableben veröffentlicht wurden. ${ }^{6}$ Trew selbst machte seine Bibliothek über die Publikation mehrerer Kataloge seines Bibliotheksbestands der Gelehrtenwelt bekannt.' Zudem wurden seine umfangreichen Sammlungen von Büchern und von ihm in Auftrag gegebene botanische Illustrationen zum Ausgangspunkt mehrerer bedeutender botanischer Tafelwerke, die Trew und seine gelehrten Freunde in Zusammenarbeit mit Nürnberger Künstlern herausgaben. ${ }^{8}$

Für die heutige Forschung sind gerade diese botanischen Illustrationen von besonderem Interesse. Darunter befinden sich 1.350 kolorierte Zeichnungen, die in Trews Auftrag u.a. von Dionysius Ehret angefertigt wurden, 838 Zeichnungen aus dem handschriftlichen

$5 \quad$ Vgl. Trews Stammbücher. Band I-V. [UBE MS 1471-1475].

6 Vgl. Fridolin DressLer: Fränkische Privatbibliotheken im Spiegel von Reiseberichten des 18. Jahrhunderts. In: Manfred von Arnim (Hg.): Festschrift Otto Schäfer. Zum 75. Geburtstag am 29. Juni 1987. Stuttgart 1987, S. 495-514, hier S. 498 f.

7 Der ersten Centurie des Blackwellschen Kräuterbuch gab Trew eine Bibliografie botanischer Standardwerke bei, die bis zum Jahr 1550 gedruckt worden waren und sich in seiner Bibliothek befanden. Außerdem veröffentlichte er 1768, ein Jahr vor seinem Tod, den ersten und einzigen Katalog seiner Büchersammlung, in dem sein Zeitschriftenbestand vorgestellt wird.

8 Vgl. z.B. Thomas Schnalke: Das genaue Bild, das schöne Bild. In: Thomas Schnalke (Hg.): Natur im Bild. Anatomie und Botanik in der Sammlung des Nürnberger Arztes Christoph Jacob Trew. Erlangen 1995 (Schriften der Universitätsbibliothek Erlangen-Nürnberg Bd. 27), S. 99-129; Richard N. Wegner: Christoph Jacob Trew (1695-1769). Ein Führer zur Blütezeit naturwissenschaftlicher Abbildungswerke in Nürnberg im 18. Jahrhundert. In: Rudolph Zaunick (Hg.): Mitteilungen zur Geschichte der Medizin der Naturwissenschaften und der Technik. Leipzig 1940 (Bd. 39, Heft 1 und 2), S. 218-228. 
Nachlass Conrad Gessners sowie 367 Vorzeichnungen zum Hortus Eystettensis. ${ }^{9}$ Trews Bibliothek ist im Gegensatz zum Großteil der frühneuzeitlichen Gelehrtenbibliotheken noch heute fast vollständig erhalten. Darüber hinaus sind zahlreiche umstehende Quellen wie Trews Korrespondenzen oder handschriftliche Bibliothekskataloge in der UB Erlangen vorhanden. ${ }^{10}$ Dies macht die Trew-Sammlungen zu einer wichtigen Quelle nicht nur für bibliotheksgeschichtliche Studien, sondern auch für medizin- bzw. wissenschaftsgeschichtliche, teils netzwerkanalytische Untersuchungen zur ärztlichen Praxis im 18. Jahrhundert sowie zur Entwicklung von Medizin und Naturkunde in der Frühen Neuzeit. ${ }^{11}$

9 Vgl. Hans-Otto Keunecke: Die Trewschen Sammlungen in Erlangen. In: Thomas Schnalke (Hg.): Natur im Bild. Anatomie und Botanik in der Sammlung des Nürnberger Arztes Christoph Jacob Trew. Erlangen 1995 (Schriften der Universitätsbibliothek Erlangen-Nürnberg Bd. 27), S. 131-166, hier S. 155 f.

$10 \quad \mathrm{Zu}$ den einzelnen Teilen von Trews Sammlung vgl. Elisabeth EnGL, Ursula RaUtenberg: Der Nürnberger Arzt und Naturforscher Christoph Jacob Trew (1695-1769) und seine Sammlungen in der Universitätsbibliothek Erlangen. In: Ursula Rautenberg (Hg.): Jahresbericht des Instituts für Buchwissenschaft an der Friedrich-Alexander-Universität Erlangen-Nürnberg 2017. Erlangen 2018, S. $13-24$.

11 Die Erlanger Tagung „Sammeln, Forschen, Publizieren. Christoph Jacob Trew im Netzwerk der frühmodernen Gelehrtengemeinschaft" (15.-17.5.2019, FAU Erlangen) hat diese verschiedenen Forschungsrichtungen zu Trew zusammengeführt. Der Tagungsband wird voraussichtlich 2021 unter dem Titel „Sammeln, Forschen und Publizieren im Netzwerk der frühmodernen Gelehrtengemeinschaft. Der Nürnberger Arzt und Naturforscher Christoph Jacob Trew (1695-1769), seine Bibliothek und ihre Bedeutung für die Botanik" bei Hiersemann erscheinen. Trews Bibliothek, deren Bestand, das Zusammentragen, Ordnen und Verzeichnen der Bücher sowie die Nutzungsfunktionen der Büchersammlung hat ENGL in ihrer Dissertation untersucht. Diese wird 2020 unter dem Titel „Die medizinisch-naturkundliche Bibliothek des Nürnberger Arztes Christoph Jacob Trew. Analyse einer Gelehrtenbibliothek im 18. Jahrhundert" im Hiersemann-Verlag in der Reihe „Bibliothek des Buchwesens” erscheinen. 
2 Gelehrter Arzt, Sammler und Forscher: kurzer biographischer Abriss

Der Apothekersohn Christoph Jacob Trew wurde am 26. April 1695 in Lauf bei Nürnberg geboren und studierte im nahen Altdorf u.a. bei Lorenz Heister und Johann Jacob Baier Medizin. ${ }^{12}$ Nach Abschluss seines Studiums ging Trew auf eine dreijährige peregrinatio academica durch die Schweiz, Frankreich, die Niederlande und deutschsprachige Gebiete. Auf dieser Reise konnte Trew seine Studien vervollständigen und bei seinen Besuchen in Bibliotheken, botanischen Gärten und anatomischen Theatern wichtige Kontakte in die Gelehrtenwelt knüpfen, bspw. zu den Pariser Botanikern Antoine und Bernard de Jussieu. ${ }^{13}$ In dieser Zeit könnte Trew auch erste Bücher für seine Bibliothek erworben haben. Angesichts seines knappen Reisebudgets, für das Trew auf ein Stipendium der Stadt Nürnberg angewiesen war, werden sich derartige Einkäufe jedoch in Grenzen gehalten haben. Nachdem sein ehemaliger Lehrer Heister ihn über eine offene Professur an seiner Alma mater informiert hatte, brach Trew seine Gelehrtenreise $a b$, um sich in der Heimat auf diese Stelle zu bewerben. Trotz der Fürsprache Heisters blieben diese Bemühungen erfolglos und Trew ließ sich im Herbst 1721 als städtischer Arzt in Nürnberg nieder. Dort heiratete er im Jahr 1723 eine reiche Witwe und kaufte sich vier Jahre später ein Haus mit Garten im Nürnberger Stadtviertel Wespennest. Als städtischer Arzt gewann er rasch an Beliebtheit und Ansehen und wurde 1734 zum Leibarzt am

12 Vgl. Thomas Schnalke: Einleitung: „Dem publico zu dienen...”. In: Thomas Schnalke (Hg.): Natur im Bild. Anatomie und Botanik in der Sammlung des Nürnberger Arztes Christoph Jacob Trew. Erlangen 1995 (Schriften der Universitätsbibliothek Erlangen-Nürnberg Bd. 27), S. 9-19, hier S. 9.

13 Vgl. Thomas Schnalke: Sammeln und Vernetzen. Christoph Jacob Trew (16951769) in seiner botanischen Matrix. In: Regina Dauser, Stefan Hächler, Michael Kempe, Franz Mauelshagen, Martin Stuber (Hg.): Wissen im Netz. Botanik und Pflanzentransfer in europäischen Korrespondenznetzen des 18. Jahrhunderts. Berlin 2008 (Colloquia Augustana Bd. 24), S. 171-200, hier S. 176-178. 
ansbachischen Hof ernannt. In der Nürnberger Ärzteschaft übernahm er zudem gegen Ende seines Lebens mehrmals hohe Ämter. ${ }^{14}$

Seine gelehrten Interessen verfolgte Trew auch als städtischer Arzt weiterhin. Studierte Ärzte grenzten sich in der Frühen Neuzeit über ihre Gelehrsamkeit von den übrigen Heilergruppen ab. 1727 wurde Trew in die renommierte kaiserliche Akademie der Naturforscher (Leopoldina) aufgenommen, deren Zeitschrift er als Director Ephemeridum ab dem Jahr 1744 herausgab. Auch in anderen internationalen Akademien wie der Royal Society in London oder der Societá Botanica in Florenz war Trew Mitglied. Über sein weitreichendes Korrespondentennetzwerk war er in der Gelehrtenwelt wohlbekannt. Ab dem Jahr 1731 war Trew Mitherausgeber des Commercium litterarium, der ersten medizinischnaturkundlichen Wochenzeitschrift im deutschsprachigen Raum, über die er zahlreiche neue Kontakte knüpfen konnte. ${ }^{16} \mathrm{Ab}$ den 1750er Jahren publizierte er mehrere Tafelwerke, die zu den Glanzstücken der Nürnberger botanischen Buchillustration gezählt werden. ${ }^{17}$ Für diese und andere Publikationen waren seine umfangreichen Sammlungen Voraussetzung. Ein Jahr vor seinem Tod am 18. Juli 1769 besaß Trew 34.000 Bücher, 16.000 Dissertationen, knapp 20.000 größtenteils antiquarische

14 Vgl. Thomas Schnalke: Christoph Jacob Trew. Laufer Stadtkind - Nürnberger Weltbürger. In: Fundgrube. Beilage der Pegnitz-Zeitung für Heimatkunde und Lokalgeschichte 36 (1995), S. 21-36, hier 22-23, 26; Schnalke: Einleitung: „Dem publico zu dienen...”, 11-12, 17; Thomas Schnalke: Ein Korb für Göttingen. Christoph Jacob Trew im Briefwechsel mit dem Chirurgen Carl Friedrich Gladbach. In: Medizinhistorisches Journal 29 (1994), S. 233-275, hier S. 263.

15 Vgl. Thomas Broman: Rethinking professionalization. Theory, practice, and professional ideology in eighteenth-century German medicine. In: The Journal of Modern History 67 (1995), S. 835-872, hier S. 841.

16 Zu dieser Zeitschrift vgl. Tilman Tassilo Rupert Rau: Das Commercium Litterarium. Die erste medizinische Wochenschrift in Deutschland und die Anfänge des medizinischen Journalismus. Bremen 2009 (Presse und Geschichte - Neue Beiträge Bd. 42).

17 Vgl. Schnalke: Sammeln und Vernetzen. Christoph Jacob Trew (1695-1769) in seiner botanischen Matrix, S. 180-181; Schnalke: Einleitung: „Dem publico zu dienen..., S. 14-16. 
Gelehrtenbriefe, ${ }^{18} 58$ Herbar-Bände mit getrockneten Pflanzen, zahlreiche gemalte und lebende Pflanzen sowie weitere Naturalien und anatomische Präparate. Diese vermachte er testamentarisch der Universität in Altdorf..$^{19}$ Von dort kam die Trew-Bibliothek nach der Auflösung der Altdorfer Universität zu Beginn des 19. Jahrhunderts an die Universitätsbibliothek in Erlangen. ${ }^{20}$

3 Die Trew-Bibliothek: Sammelinteresse, Bibliotheksbestand und BuCHBESCHAFFUnG

Trews Sammelinteresse und Bibliotheksbestand

Den Grundstock von Trews Bibliothek bildeten die Sammlungen von seinem Großvater Abdias (1597-1669), Astronom und Mathematiker, seinem Großonkel, dem Pfarrer Johann Georg (1604-1669), sowie seinem Vater, dem Apotheker Christoph (1641-1717). Wie groß diese Sammlungen waren, ist nicht bekannt. Trew baute diese im Lauf seines Lebens kontinuierlich aus. ${ }^{21}$ Er interessierte sich vorwiegend für medizinisch-naturkundliche Titel, die für ihn als praktizierenden Arzt

18 Trew sammelte 13.500 antiquarische Gelehrtenbriefe, in denen die Gelehrten ihre neuen, teils nie publizierten Erkenntnisse diskutierten. Darüber hinaus haben sich 4.720 Briefe an Trew sowie 852 Briefe bzw. Briefentwürfe Trews erhalten. Vgl. Eleonore Schmidt-HerRLing: Die Briefsammlung des Nürnberger Arztes Christoph Jacob Trew (1695-1769) in der Universitätsbibliothek Erlangen. Erlangen 1940 (Katalog der Handschriften der Universitätsbibliothek Erlangen Bd. 5) Sämtliche Briefe wurden von der UB Erlangen digitalisiert und sind über deren OPAC frei zugänglich.

19 Vgl. Eleonore Schmidt-Herrling: Die Bibliothek des Arztes Christoph Jacob Trew. In: Gunda Werner, Eleonore Schmidt-Herring (Hg.): Die Bibliotheken der Universität Altdorf. 69. Beiheft zum Zentralblatt für Bibliothekswesen. Leipzig 1937, S. 88-138, hier S. 123; Christoph Jacob Trew: Catalogvs bibliothecae medicae philosophicae et miscellaneae. Decursu quinquaginta annorum In privatum et publicum usum collectae ac porro ... Augendae. Norimbergae [1768]; Extract concepti donationis 18.2.1768. [UBE A.U.A 181], S. lr-2r.

20 Zur Trew-Bibliothek an der UB Erlangen vgl. ausführlich Keunecke: Die Trewschen Sammlungen in Erlangen (Anm. 9.)

21 Vgl. Schmidt-Herrling: Die Bibliothek des Arztes Christoph Jacob Trew, S. 97-98. 
und gelehrten Botaniker relevant waren. Zunächst war Trew bestrebt, die Standardwerke aus diesen beiden Bereichen zusammenzutragen. ${ }^{22}$ Spätestens zu Beginn der 1750er Jahre, nach seiner Ernennung zum director ephemeridum und seiner Aufnahme in weitere prestigeträchtige Akademien, erweiterte er sein Sammelinteresse signifikant: „Ich sammle alle mir noch fehlenden Schriften, die zur Arzney und Naturwissenschaft gehören, sie mögen gut oder schlecht sein, wenigstens historiae caussa, weil meine Sammlung, wenn Gott will, zu einem künftigen öffentlichen gemeinen Nutzen bestimmt ist." ${ }^{23}$ Trew wollte der umfassenden Bestandsaufnahme der vorhandenen Literatur wegen sowohl zeitgenössisch wertgeschätzte als auch schlecht angesehene Bücher sammeln. Jeder Text wurde als potentiell wertvoll für die gelehrte Arbeit bzw. die Bestandsaufnahme der bisherigen gelehrten Publikationen und damit als sammelnswert angesehen. Die Bibliothek sollte zudem für ihre spätere öffentliche Zugänglichmachung in Altdorf möglichst vollständig sein. Eine Büchersammlung, die zumindest in Teilbereichen vollständig war, wurde zeitgenössisch als Ideal angesehen. ${ }^{24}$

Eine erste genauere Beschreibung des Bestands liegt aus der Mitte des Jahres 1752 vor. Trew schickte einen gedruckten, publizierten Katalog der in seiner Bibliothek vorhandenen botanischen Standardwerke aus der Frühzeit des Buchdrucks an seinen Korrespondenten Albrecht von Haller und erklärte in dem beigelegten Brief:

„Ein großes Vergnügen werde ich nun haben, wenn ich meinen seit 30 Jahren gesammelten Vorrat von medizinischen, physikalischen, philosophischen u[nd] mathematischen Büchern (deren Zahl auctoris jedes Traktat besonders gerechnet, nun über 12000 sich erstreckt. Kleine piecem von 3 oder 4 Bögen gegen 3000, und dissert[ationes] acad[emicarum]

Vgl. Elisabeth EngL: Die medizinisch-naturkundliche Bibliothek des Nürnberger Arztes Christoph Jacob Trew. Analyse einer Gelehrtenbibliothek im 18. Jahrhundert. Stuttgart 2020, Kapitel IV.4.

23 Vgl. Brief von Popowitsch, Johann Siegmund Valentin an Trew, Christoph Jacob, Briefnr. 115 vom 15.10.1763, S. 1r.

24 Vgl. Brief von Ludwig, Christian Gottlieb an Trew, Christoph Jacob, Briefnr. 51 vom 29.11.1755, S. 2r. 
über 10000 nicht mit gezählt) perlustrieren und anzeigen könnte, sollte es auch nur auf gleiche Weise, wie in diesem specimine, geschehen."25

$\mathrm{Zu}$ Beginn der 1750er Jahre besaß Trew bereits einen beachtlichen Bibliotheksbestand, den er jedoch noch deutlich erweitern wollte. ${ }^{26}$ Ein Jahr später war seine Sammlung so weit angewachsen, dass er seinen Bibliotheksraum umbauen musste. Über ein halbes Jahr hinweg wurde im zweiten Stock seines Hauses ein größeres Zimmer für seine Büchersammlung hergerichtet. ${ }^{27}$ Dieser Umbau scheint Trews Sammeleifer noch verstärkt zu haben, denn Mitte des Jahres 1757 befanden sich bereits 20.000 Titel in seiner Bibliothek..$^{28}$ Bis November 1762 wurde die Sammlung rasch um weitere 10.000 Titel vermehrt, zu denen bis zu Trews Tod im Jahr 1769 nur noch wenige Titel hinzukamen. ${ }^{29}$

Trews im engeren Sinne medizinisch-naturkundlicher Kernbestand, der noch heute geschlossen nach Trews originalen Signaturen im Magazin der UB Erlangen aufgestellt ist, umfasst insgesamt 15.051 Titel. ${ }^{30}$ Die übrigen, quantitativ nicht genau erfassbaren Titel komplettieren diesen Kernbestand und lassen sich in vier breiter gefasste Bereiche der Medizin und Naturwissenschaften, der klassischen Bildung

25 Vgl. Brief von Trew, Christoph Jacob an Haller, Albrecht von, Briefnr. 303 vom 7.1752, S. lv.

26 Vgl. Brief von Trew, Christoph Jacob an Creutznach, Leonhard Stephan von, Briefnr. 181 vom 16.10.1752, lr.

27 Vgl. Brief von Trew, Christoph Jacob an Cox, H. Shute, Briefnr. 170 vom 11.1753, S. lr; vgl. Brief von Trew, Christoph Jacob an Cox, H. Shute, Briefnr. 171 vom 5.12.1753, S. lr.

28 Vgl. Brief von Trew, Christoph Jacob an Watzdorff, Eleonora Augustina von, Briefnr. 796 vom 2.9.1757, S. lv.

29 Vgl. Brief von Trew, Christoph Jacob an Kadelbach, Christian Friedrich, Briefnr. 409 vom 11.1762, S. lv.

30 Für die folgende Auswertung von Trews Bibliotheksbestand vgl. EngL: Die medizinisch-naturkundliche Bibliothek des Nürnberger Arztes Christoph Jacob Trew, Kapitel IV.l. 
eines gelehrten Arztes, der Literärgeschichte sowie der Miscellanea einordnen. ${ }^{31}$

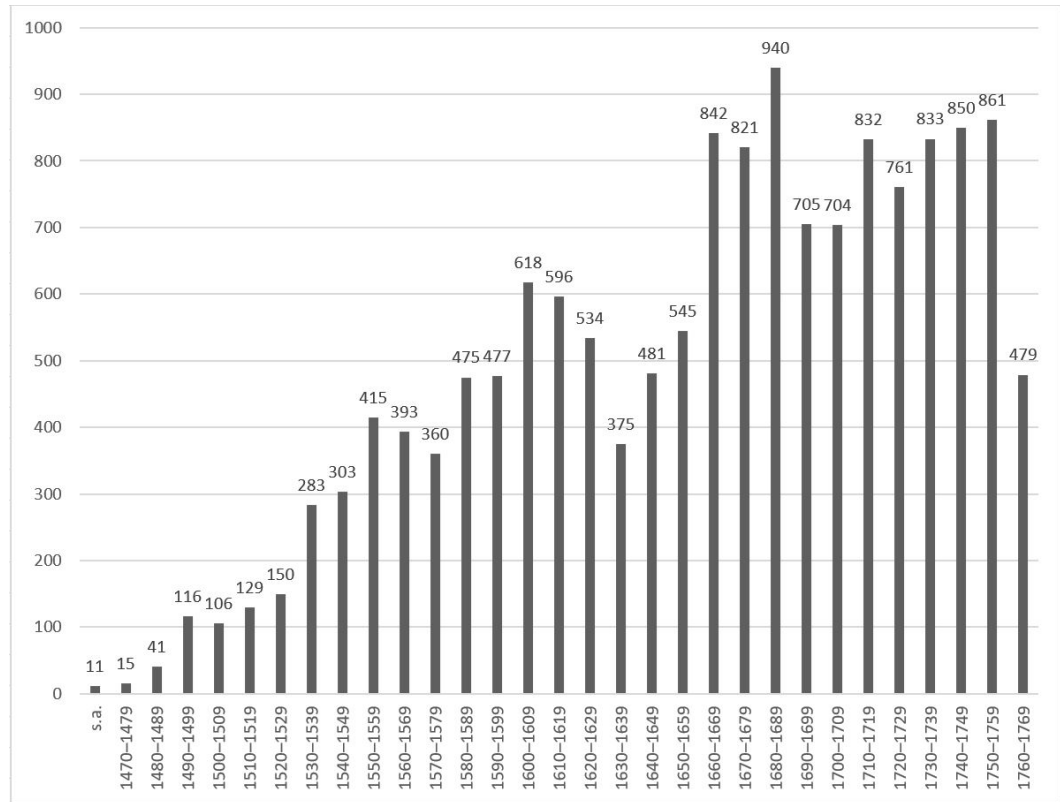

"Titel in Trews medizinisch-naturkundlichem Kernbestand nach Publikationsjahren in absoluten Zahlen"

Die obenstehende Abbildung zeigt die Verteilung der Erscheinungsjahre in Trews Kernbestand. 8.149 Titel, über die Hälfte des Kernbestands, wurde in einem Zeitraum von etwa 100 Jahren vor Trews Tod gedruckt. 3.091 Titel liegen aus dem 16. Jahrhundert und 172 Titel aus dem 15. Jahrhundert vor. ${ }^{32}$ Das Verhältnis von älteren und neueren Werken ähnelt den Beständen anderer medizinisch-naturkundlicher

$31 \quad$ Die Trew-Titel außerhalb des Kernbestands sind heute nur teilweise noch geschlossen nach Trews Signaturen aufgestellt. Im OPAC der UB Erlangen ist nicht bei allen Exemplaren ihre Trew-Provenienz angegeben, sodass nur ein Teil dieser Titel über den OPAC ermittelt werden kann. Weitere elf Titel sind undatiert. 
Sammlungen. Doch die absolute Anzahl an Frühdrucken ebenso wie die Gesamtzahl von Titeln in Trews Besitz ist außergewöhnlich hoch. Gedruckt wurden die Titel vorwiegend im deutschsprachigen Raum, daneben liegen insbesondere aus den großen Buchzentren Westeuropas wie Paris, Amsterdam und Leiden zahlreiche Bücher vor.

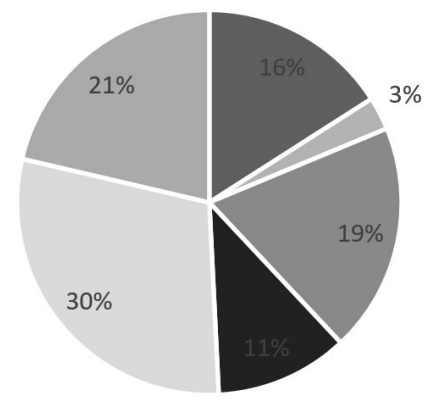

- Anatomie

- Allgemeine Therapie

Naturkunde
Pathologie

- Spezielle Pathologie/Therapie

Weitere medizinische Schriften

„Prozentuale Verteilung der Titel der sechs inhaltlichen Gruppen in Trews Kernbestand"

Inhaltlich lässt sich fast ein Drittel der Titel der Naturkunde mit einem Schwerpunkt auf Chymie $^{33}$ und allgemeinen Werken zur Naturkunde zurechnen. Zudem sind viele Bücher zur Anatomie als Leitdisziplin der Medizin und der Allgemeinen Therapie, besonders zur Materia medica und der Pharmakologie, vorhanden. Zur Speziellen Pathologie und

33 Die Begriffe Alchemie und Chemie beziehungsweise Chemiatrie wurden bis zum Ende des 17. Jahrhunderts synonym verwendet. Der zeitgenössische Begriff Chymie kann als Oberbegriff für Alchemie und Chemie in der Frühen Neuzeit verwendet werden. Vgl. William R. Newman: From alchemy to "chymistry”. In: Katharine PARK, Lorraine Daston (Hg.): The Cambridge history of science. Early modern science. Cambridge 2006, S. 497-517, hier S. 497-499. 
Therapie zu bestimmten Gebrechen sowie zur Pathologie, die sich erst gegen Ende des 18. Jahrhunderts als eigenständiger Bereich der Medizin herausbildete, besaß Trew demgegenüber deutlich weniger Titel. Die übrigen $21 \%$ der Titel verteilen sich auf Standard- bzw. Referenzwerke sowie antike bzw. arabisch-islamische Bücher, die größtenteils in lateinischen Übersetzungen vorliegen.

Trew baute va. im Bereich der Allgemeinen Anatomie, Therapie und Naturkunde sowie in der Chymie eine annähernd vollständige medizinisch-naturkundliche Bibliothek auf, die erwartungsgemäß einen Schwerpunkt auf dem deutschsprachigen Raum zeigt. Insbesondere die englischen Autoren, deren Werke in der Frühen Neuzeit auf dem Kontinent nur schwer erhältlich waren, sowie in geringerem Maße die im 18. Jahrhundert führenden niederländischen Ärzte sind in Trews Büchersammlung unterrepräsentiert.

Trews Vollständigkeitsanspruch ist für die älteren und neueren Titel in seiner Bibliothek differenziert zu betrachten. Für die Publikationen des 17. und 18. Jahrhunderts bezieht sich dieser v.a. auf Werke bekannter wie unbekannter Autoren, während er für Publikationen des 15. und 16. Jahrhunderts eher Ausgaben der bedeutenden Standardwerke bezeichnet.

Trew wollte sämtliche zeitgenössischen, inhaltlich zu seiner Sammlung passenden Werke besitzen. Diese sollten möglichst in jeweils der Ausgabe vorliegen, die sowohl inhaltlich als auch bezüglich ihrer Ausstattung für die gelehrte Arbeit am besten geeignet eingeschätzt wurde. Bei Werken des 17. und 18. Jahrhunderts war dies in der Regel die neueste Ausgabe. Dadurch sollte sämtliches medizinisch-botanisches Wissen auf dem Stand der Zeit in Trews Bibliothek versammelt werden. Für die frühen Publikationen des 15. und 16. Jahrhunderts ist eine derartige Sammlung in der Praxis noch deutlich schwerer umzusetzen gewesen als für diese späteren Drucke. Zudem wäre sie wenig hilfreich, da diese Werke im 18. Jahrhundert größtenteils als veraltet angesehen wurden. Eine Beschränkung auf die zu Trews Zeit als bedeutend eingeschätzten, weiterhin häufig zitierten Werke konnte daher aus zeitgenössischer Sicht den Wissensstand des 15. und 16. Jahrhunderts ebenfalls vollständig 
abbilden. Die Ausweitung des Sammelanspruchs bei frühen Drucken auf die Ausgabenebene liegt in Trews buchkundlichen Interessen begründet und ist als Besonderheit zu sehen, die seine Bibliothek auch von anderen großen Büchersammlungen der Frühen Neuzeit abhob. Ein Vergleich von Trews Bestand an ausgewählten botanischen Standardwerken mit Albrecht von Hallers Büchersammlung zeigt, dass diese Werke zwar fast alle in den Bibliotheken der beiden gelehrten Ärzte vorhanden sind. Haller besaß jedoch jedes Werk in nur ein oder zwei Ausgaben, während Trew 60\% der laut Universal Short Title Catalogue ${ }^{34}$ insgesamt publizierten Auflagen zusammentrug. Sofern sich eine gute Gelegenheit bot, kaufte Trew darüber hinaus einige Titel, die über seinen Sammelanspruch hinausgingen. Diese sah er als fakultative Ergänzung seiner Sammlung an. Insgesamt zeichnet sich Trews Bibliothek durch das angestrebte Ziel der Vollständigkeit aus, das er annähernd erreichte.

\section{Zusammentragen der Bücher für die Bibliothek}

Die hohen Kosten seiner umfangreichen Sammeltätigkeit versuchte Trew auf mehrere Weisen zu senken und zu kontrollieren. So verzichtete er auf die Anstellung mehrerer Helfer, die ihn bei der Organisation der Büchersammlung hätten unterstützen können. Neben dem gelehrten Arzt Gustav Philipp Zwinger, der Trew spätestens ab dem Jahr 1762 unterstützte, scheint Trew lediglich zeitweise, für die wiederholte Erstellung neuer Bibliothekskataloge, weitere amanuenses beschäftigt zu haben. ${ }^{35}$ Außerdem ließ Trew die teils gebraucht bei Auktionen erworbenen Bücher direkt in seiner Bibliothek aufstellen, anstatt diese aufwändig und kostspielig neu binden zu lassen. Eínheitliche

$34 \quad$ Vgl. https://www.ustc.ac.uk/explore [10.12.2019].

35 Vgl. Schmidt-Herrling: Die Bibliothek des Arztes Christoph Jacob Trew, S. 113; Brief von Trew, Christoph Jacob an Popowitsch, Johann Siegmund Valentin, Briefnr. 627 vom 22.4.1762, S. 1r; Keunecke: Die Trewschen Sammlungen in Erlangen, S. 144-145; Brief von Trew, Christoph Jacob an Haller, Albrecht von, Briefnr. Reinschrift, Entwurf bei Trew Briefnr. 306 vom 6.1.1757, S. 1r. 
Bucheinbände waren besonders bei adligen Sammlern beliebt, da diese zu einem repräsentativen Gesamteindruck der Bibliothek beitrugen. ${ }^{36}$

Darüber hinaus scheint sich Trew für seine Bücherkäufe zumindest grobe Obergrenzen gesetzt $\mathrm{zu}$ haben, um sich finanziell nicht $\mathrm{zu}$ verausgaben. Dies betrifft sowohl einzelne Käufe als auch Erwerbungen über einen längeren Zeitraum hinweg. ${ }^{37}$ Der Leipziger Medizinprofessor Christian Gottlieb Ludwig, ein Korrespondent Trews, legte bspw. einen Jahresetat für seine Bibliotheksausgaben fest - auch wenn er diesen bisweilen deutlich überschritt. ${ }^{38}$

Die tiefgehenden buchhändlerischen und gelehrten Bücherkenntnisse, die für den Aufbau einer großen Bibliothek benötigt wurden, konnte Trew aus Korrespondenzen mit Buchhändlern sowie buchhändlerischen Katalogen und weiteren Bücherverzeichnissen wie Bibliografien oder gelehrten Zeitschriften ziehen. Die Nutzung dieser Informationsmittel war zeitgenössisch unter Gelehrten weit verbreitet. Trew besaß über 336 verschiedenartige Bücherkataloge und 389 gelehrte Zeitschriften, die er geschlossen in seiner Bibliothek aufstellte, um bei Bedarf rasch darauf zugreifen zu können. ${ }^{39}$

Von besonderer Bedeutung waren zudem seine gelehrten Korrespondenzpartner. Diese konnten wie Trew neben den oben genannten Informationsquellen auf gelehrte Publikationen sowie das Wissen ihrer eigenen gelehrten Freunde zurückgreifen, die sich ebenfalls über Jahre hinweg umfassendes Bücherwissen angeeignet

36 Vgl. Kristian Jensen: Creating a better past. Collectors of incunabula in the late eighteenth century. In: Bettina Wagner (Hg.): Early printed books as material objects. Berlin [u.a.] 2010 (IFLA publications Bd. 149), S. 281-290, hier S. 283.

37 Vgl. Brief von Trew, Christoph Jacob an Popowitsch, Johann Siegmund Valentin, Briefnr. 613 vom 19.2.1759, S. lv.

38 In einem Brief an Trew, in dem Ludwig die geplante inhaltliche Neuausrichtung seiner Bibliothek beschreibt, muss dieser zugeben: „Dieses Jahr bin ich im Bücher kaufen zu weit gegangen, und habe das Triplum von meinem jährlich aufzuwenden Bibliotheksgeld vertan, man muss allemal viel auf res domestides sehen." Ludwig (wie Anm. 23), S. 2r.

39 Vgl. Trews Bandkataloge sowie TREw: Catalogus bibliothecae medicae philosophicae et miscellaneae. 
hatten. Über die von den Gelehrten ausgebildeten Netzwerke konnten diese Informationen gesammelt und im Rahmen der gelehrten Freundschaften ausgetauscht werden. Wie hilfreich die Hinweise der einzelnen Korrespondenzpartner für Trews Wissen über den Buchmarkt sein konnten, war hauptsächlich von den folgenden Faktoren abhängig: Die Einbindung einer Person in die Gelehrtenrepublik bestimmte über die ihr zur Verfügung stehenden Informationsquellen sowie darüber, ob lediglich buchhändlerische oder auch gelehrte Bücherkenntnisse weitergegeben werden konnten. Auf dem Überschneidungsgrad der Interessensgebiete der beiden Korrespondenzpartner beruhten zudem Umfang und Relevanz der von den zwei Kommunikationspartnern gesammelten und an den jeweils anderen weitergegebenen Informationen. Darüber hinaus hatte der Wohnort einer Person Einfluss darauf, welche Informationsquellen ihr zugänglich waren. Besonders hilfreich konnte es sein, wenn ein gelehrter Freund gute, geografisch weit gestreute Kontakte in die Gelehrtenwelt sowie zu Händlern oder ungelehrten Mittelsmännern unterhielt. Besonders wertvoll war dieses Kontaktnetz, wenn sich dieses deutlich von dem eigenen Netzwerk unterschied und gleichzeitig zahlreiche Personen einband, die ihrer ähnlichen Interessen wegen Informationen zu den gleichen Themenfeldern sammelten. In ihrem Verbund konnten die Korrespondenten und die weiteren Informationsmittel einen Überblick über den europäischen Buchmarkt medizinischer und naturkundlicher Titel geben. Aus diesem konstituierte sich Trews Sammelfeld, sodass diese Kenntnis Voraussetzung für das Zusammentragen seines Bibliotheksbestands war.

Der Kauf der Bücher erforderte v.a. große organisatorische Leistungen, Kenntnisse über die Strukturen des Buchmarkts sowie ein potentes Kontaktnetzwerk. Nur an seinem Wohnort Nürnberg konnte Trew selbst Geschäfte bei Buchhändlern tätigen. Bei den Kaufvorgängen in anderen Städten musste er darauf vertrauen, dass seine Aufträge von den angeschriebenen Buchhändlern oder Mittelsmännern in seinem Sinne ausgeführt wurden. Die einzelnen Kaufaufträge wurden jeweils an diejenigen Personen vergeben, denen aufgrund ihres Wohnorts 
oder ihrer Kontakte die größtmöglichen Erfolgschancen für deren Ausführung eingeräumt werden konnten. Von größter Bedeutung für den Kauf der Bücher waren Bücherauktionen, die als kostengünstigere Alternative zu der Buchbeschaffung über Sortimenter angesehen wurden. Bei einer einzigen Versteigerung ließ Trew teilweise Bücher im niedrigen dreistelligen Bereich kaufen. ${ }^{40}$ Den billigeren und einfacheren, zeitsparenden Weg des geschlossenen Aufkaufs von Bibliotheken auf dem Gebrauchtbuchmarkt, der jedoch dem planvollen Aufbau einer Sammlung unter weitestgehender Vermeidung von Doubletten entgegensteht, hat Trew nicht gewählt.

\section{Ordnung der Bücher in den Bibliothekskatalogen und im Bibliotheksraum}

Um die zusammengetragenen Bücher nutzen zu können, mussten diese mit Blick auf die intendierten Nutzungssituationen geordnet im Bibliotheksraum aufgestellt bzw. in den Katalogen verzeichnet werden. Bereits Gabriel Naudé hatte in seinem Advis pour dresser une bibliothèque eine ungeordnete Büchersammlung als vollkommen unbrauchbar bezeichnet. ${ }^{41}$ In der UB Erlangen sind vier zeitgenössische Kataloge von Trews Büchersammlung erhalten: ein fünfbändiger Standortkatalog, ein siebenbändiger Autorenkatalog, ein ungebundener und unvollständiger systematischer Katalog sowie ein ebenfalls ungebundener Katalog, der die Folio- und einen Teil der Quartbände verzeichnet. ${ }^{42}$

40 So kaufte Trew bspw. 117 Bücher aus der Bibliothek von Johann Friedrich Christ und 118 Bücher aus dem Nachlass von Joseph von Rathgeb. Vgl. Brief von Ludwig, Christian Gottlieb an Trew, Christoph Jacob, Briefnr. Beilage e.; Brief von Popowitsch, Johann Siegmund Valentin an Trew, Christoph Jacob, Briefnr. 88 vom 13.5.1759.

41 Vgl. Gabriel Naudé: Advis pour dresser une bibliothèque. Neudruck 1963, Leipzig 1627, S. 97-98.

42 Trews Autorenkatalog. Band I-VII. [UBE MS 2474-2480]; Trews Standortkatalog. Band I-V. [UBE MS 2481-2485]; Trews Katalog der Foliobände. Band I-III. [UBE MS 2494 I-III]. Neben diesen vier Katalogen besaß Trew einen Katalog zu den Schediasmata - Kleindrucken -, sowie fünf Katalogbände zu den Dissertationen in seinem Besitz. Vgl. dazu Elisabeth Engl, Ursula Rautenberg: Der Nürnberger Arzt und Naturforscher Christoph Jacob Trew. (Anm. 10.) 
Abgesehen von dem systematischen Katalog wurden Trews Bibliothekskataloge auf einer nachgeordneten Ebene nach ähnlichen Prinzipien der Ordnung und Verzeichnung angelegt, wobei die Bücher nach inhaltlichen und formalen Kriterien klassifiziert wurden. Die Bände bzw. Titel wurden vom Allgemeinen, Älteren zum Speziellen, Neueren hin, mit den Originalwerken vor den zugehörigen Kommentaren und Übersetzungen im Regal aufgestellt und im Katalog verzeichnet. Außerdem wurden die einzelnen Bände einer Reihe ebenso geschlossen aneinandergereiht wie die chronologisch und nach Sprachen sortierten Drucke eines Werks, sodass sich eine Ausgabenchronologie ergibt.

Die Aufstellung und Ordnung der Bände im Bibliotheksraum und der Titel in Trews Bibliothekskatalogen bewegt sich im Spannungsfeld der theoretisch ausgearbeiteten Ordnungsprinzipien und deren praktischer Umsetzbarkeit im Rahmen der innenarchitektonischen Gegebenheiten sowie des stetigen Ausbaus der Bibliothek. Daher zeigen sich wie in anderen frühneuzeitlichen Bibliotheken eher Akkumulationen komplex geordneter Titel, als dass die Büchersammlung vollständig und konsequent nach tiefgehenden Ordnungsprinzipien aufgestellt und verzeichnet worden wäre. Die von Trew gewählten Prinzipien, nach denen er seine Sammlung auf der nachgeordneten Ebene zu ordnen versuchte, kommen der von Gabriel Naudé in seinem Advis empfohlenen Bibliotheksordnung sehr nahe. ${ }^{43}$

Die Einträge in Trews Bibliothekskatalogen sind nach dem Titelblatt angefertigt und enthalten sehr große Teile des Buchtitels sowie ggf. exemplarspezifische Merkmale. Am ausführlichsten sind die Titelaufnahmen im Verzeichnis der Folio- und Quartbände angelegt, in dem sämtliche Titelblattinformationen eines Drucks exakt übernommen wurden. Darüber hinaus werden die verzeichneten Titel auch durch summarische Inhaltsangaben grob erschlossen. Die Titelaufnahmen erlauben die Differenzierung der Sammelobjekte auf Auflagenebene, die sowohl für deren Beschaffung als auch Nutzung grundlegend ist. Bibliothekskataloge in der UB Erlangen. 
In Ansätzen ermöglichen sie außerdem die für die gelehrte Nutzung erforderliche Beurteilung einzelner Werke auf Ausgabenebene bzw. die Identifizierung der für den jeweiligen Zweck am besten geeigneten Ausgabe. Insbesondere in Hinblick auf Trews umfassenden Sammelbegriff, der sich in Teilbereichen auch auf die Ausgabenebene erstreckt, waren derartig präzise Titelaufnahmen notwendig. Die umfangreichen Kataloge sind repräsentativer Ausdruck von Trews Fähigkeit zur Beherrschung der von ihm zusammengetragenen Büchermengen. ${ }^{44}$

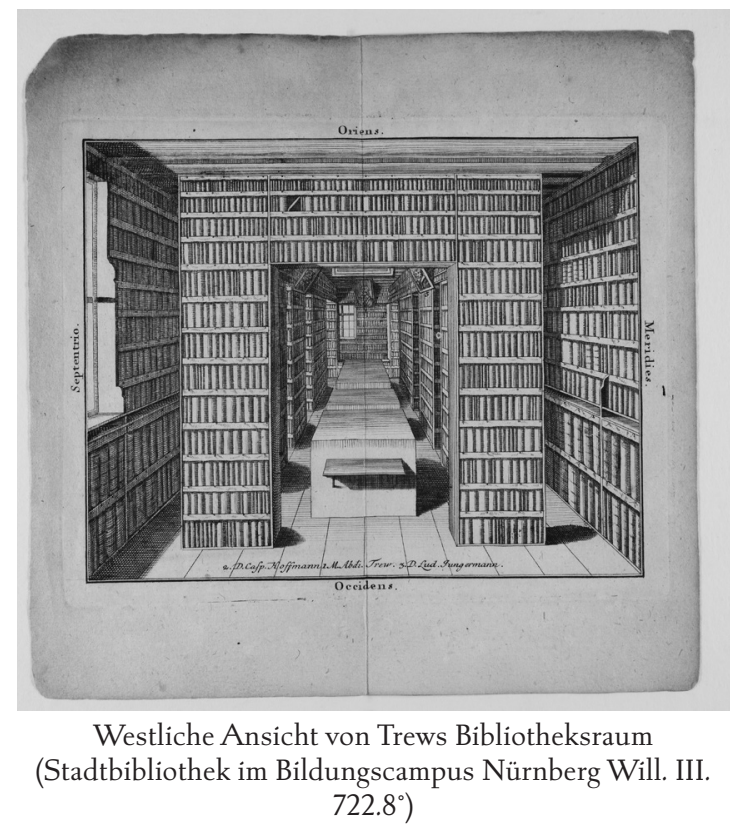

Vgl. Ulrich Johannes Schneider: Bücher und Bewegung in der Bibliothek von Herzog August. In: Frank Büttner, Markus Friedrich, Helmut Zedelmaier (Hg.): Sammeln, Ordnen, Veranschaulichen. Zur Wissenskompilatorik in der Frühen Neuzeit. Münster 2003 (Pluralisierung \& Autorität Bd. 2), S. 111-127, hier S. 113. 
Dies zeigen auch die vier Kupferstiche des Bibliotheksraums, die diesen aus vier Perspektiven darstellen und von Trew um 1760 bei dem Nürnberger Maler Johann Christoph Keller und dem Kupferstecher Johann Michael Stock in Auftrag gegeben wurden. ${ }^{45}$ Die darin erkennbare Aufstellung der Bücher und Ausgestaltung des Bibliotheksraums zeigen starke Parallelen zu den von Naudé beschriebenen Idealvorstellungen einer Bibliothek. ${ }^{46}$ Der ansonsten schmucklose, nach Osten hin ausgerichtete, helle Raum wird von den Büchern vollständig ausgefüllt. Diese wurden nach der exakten Buchhöhe in die Regale einsortiert, sodass sie wie Wandelemente wirken. Trew ging mit den 44 Gruppen, denen er die Bände ihrer Größe nach zuordnete, deutlich über die zu seiner Zeit übliche Sortierung nach Formaten hinaus. Diese Vorgehensweise erschwerte die Umsetzung der von Naudé propagierten Ordnungsprinzipien, ermöglichte jedoch eine platzsparende und optisch ansprechende Aufbewahrung der Bücher. Inwieweit die Kupferstiche des Bibliotheksraums der Wirklichkeit entsprechen, oder aus Repräsentationsgründen den zeitgenössischen Idealen angepasst wurden, kann nicht abschließend geklärt werden. Die Bilder dürften Trews Sammlung so zeigen, wie Trew seine außergewöhnlich große Bibliothek gesehen haben wollte: als umfassende und gleichzeitig geordnete Menge an Büchern.

4 Habitus und Feld: Trews Positionierung in der gelehrten Welt UND SEINE STRATEGIEN IM ÖKONOMISCHEN, SOZIALEN UND KULTURELLEN FELD

Abschließend soll der Versuch stehen, Trews zeitgenössische Bedeutung und seine vielfältigen gelehrten Interessen und Anstrengungen mithilfe der Feldtheorie des Soziologen Pierre Bourdieu ${ }^{47}$ kurz einzuordnen. Die umfangreiche Monographie von Elisabeth Engl, die zahlreiche Quellen

47 Vgl. bes. Markus Schwingel: Pierre Bourdieu zur Einführung. Hamburg, 7. ergänzte Auflage 2011, S. 59-102. 
und einen Teil der Briefschaften ausgewertet hat, bietet aufschlussreiches Material zur Trewschen Ökonomie des Sammelns und zur Bedeutung, die er seinen Sammlungen im Rahmen der Gelehrtenrepublik beimaß, sowie über seine Einbindung und Positionierung in den Netzwerken der Gelehrtenrepublik. Die folgenden Ausführungen zum Habitus als „Dispositionssystem”48 des sozialen Akteurs Trew und seines Agierens auf sozialen Feldern sollen keinesfalls die empirische und hermeneutische Forschung der letzten Jahre in Frage stellen. Allerdings scheint es sinnvoll zu sein, die nun vorliegenden reichen Ergebnisse der Trew-Forschungen, die aus unterschiedlichen disziplinären Kontexten beigetragen worden sind, zu einem Bild des Gelehrten Trew in groben Strichen zusammenzuführen.

\section{Habitus und soziale Klasse}

Trew gehörte wie sein Großvater Abdias, der Professor für Mathematik und Physik an der Universität Altdorf bei Nürnberg war, und wie auch sein Vater Christoph, Apotheker in Nürnberg, zum akademisch gebildeten Stadtbürgertum. Damit war sein Platz in der vertikal wenig durchlässigen Ständegesellschaft Nürnbergs in der ersten Hälfte des 18. Jahrhunderts festgelegt. Trews Habitus speist sich einerseits aus der familialen akademischen Tradition, er verstand es aber auch - wie die Ausführungen im zweiten und dritten Kapitel dieses Beitrags gezeigt haben - seine horizontale gesellschaftliche Mobilität gegenüber dem von Großvater und Vater Erreichten erheblich zu erweitern. Am Ende seines Lebens ist Trew ein weithin geachteter Wissenschaftler, der über seine ausgreifenden Aktivitäten soziales, intellektuelles und ökonomisches, letztendlich symbolisches Kapitel angesammelt hat. als „System dauerhafter Dispositionen [...], in dem Wahrnehmungs-, Denkund Handlungsschemata zusammenwirken"; Schwingel: Pierre Bourdieu zur Einführung, S. 65. 


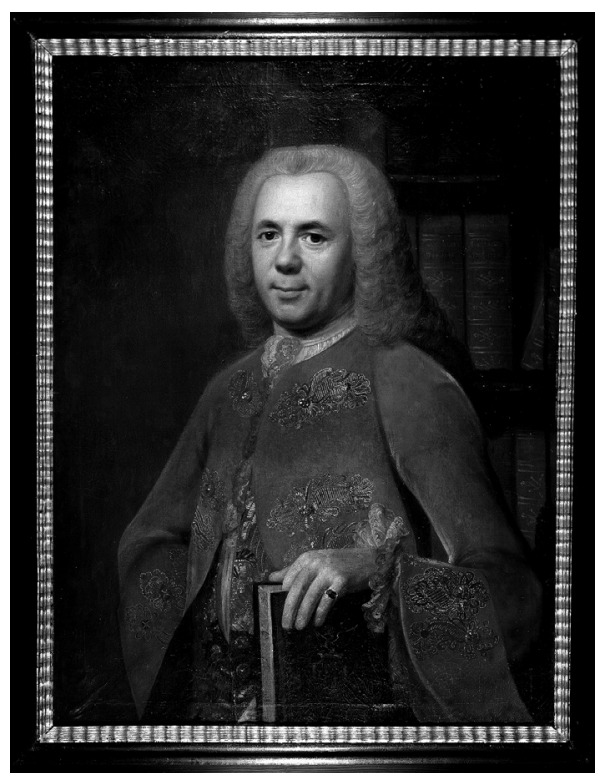

Porträt von Dominicus van der Smissen, Öl auf Leinwand, 1748 (FAU Kunstinventar Nr. 447)

Das 1748 von Dominicus van der Smissen angefertigte Porträt zeigt ihn, im Alter von 43 Jahren mit weiß gepuderter Perücke und in eine goldbestickte rote Robe gehüllt, in der geläufigen Pose des Gelehrten vor einem Bücherregal. Mit dem Zeigefinger markiert Trew eine Stelle im zugeschlagenen Buch. Es handelt sich um einen Band der Transaktionen der Kaiserlichen Akademie der Naturforscher „Leopoldina”, deren Wappen, für den Betrachter frontal sichtbar, auf dem hinteren Einbanddeckel eingeprägt ist. Das Porträt zeigt einen Mann in den „besten Jahren”, im Habitus des anerkannten Gelehrten und Mitglied bedeutender wissenschaftlicher Gesellschaften. Zitiert wird das kulturelle Kapital des Porträtierten: im Besitz von Buch und Bibliothek, institutionalisiert in den diskreten Verweisen auf die beiden bedeutenden wissenschaftlichen Gesellschaften, verinnerlicht als Praktik des gelehrten Lesens. Ökonomisches Kapitel ist, inkorporiert in der Bibliothek und transformiert in kulturelles Kapital sichtbar, aber auch an den reichen 
Gewändern. Lediglich die lebensnahe Physiognomie individualisiert das repräsentative Gelehrtenporträt, nichts deutet auf Trews fachliche Kompetenz als Botaniker und praktischer Arzt. Die Bildaussage der Darstellung Trews im Habitus des Gelehrten konzentriert sich auf das von ihm akkumulierte soziale Kapital gesellschaftlicher Ankerkennung und Wertschätzung. Dieses Porträt war für den Bibliotheksraum in Trews Nürnberger Haus gedacht. Dort hing es gegenüber dem seines Onkels Abdias. ${ }^{49}$

Während van der Smissen ein konventionelles, den ikonographischen Traditionen folgendes Auftragsporträt ablieferte, geben die Exlibris, die Trew für seine Bücher anfertigen ließ, einen genaueren Aufschluss über Trews Habitus-Konzept. Trew folgt mit dieser Praktik einem eingeübten Denk- und Handlungsschema: das Eignerzeichen kennzeichnet nicht nur den Moment der Inbesitznahme des Buchs, sondern ist mit dem Namen oder der Persönlichkeit des Eigners „sprechend” verbunden und bleibt im besten Fall bei Besitzerwechsel und über den Tod des Sammlers hinaus mit dem Buch bestehen. Ab der Mitte der 1720er Jahre dürfte Trew systematisch Bücher für seinen Bibliotheksplan gekauft haben; die lebenslange Sammeltätigkeit begleiten vier verschiedene Exlibris-Motive, die zu zwölf Varianten zusammengefügt wurden. ${ }^{50}$ Besonders repräsentativ ist ein dreiteiliges Exlibris. Bei zweien handelt es sich um sprechende Motive, die auf den Familiennamen "Trew” anspielen: Familienwappen und Hunde-Exlibris. Das Motiv des Familienwappens zeigt ein Schild, der am oberen Rand in Helm und Helmzier ausufert. Eine kleine, frei auf dem Helm stehende Figur hält ein großes Füllhorn mit Blüten und Früchten im Arm. Vgl. EngL: Die medizinisch-naturkundliche Bibliothek des Nürnberger Arztes Christoph Jacob Trew, Kapitel VI.2.2.

50 Vgl. Schmidt-Herrling: Die Bibliothek des Arztes Christoph Jacob Trew, S. 116; Vgl. Albert Treier: Das Exlibris in der Leopoldina. 104 alte deutsche Bucheigenzeichen. Schweinfurt 1955, S. 28-30. Ausführlich EngL: Die medizinisch-naturkundliche Bibliothek des Nürnberger Arztes Christoph Jacob Trew, 2010, Kapitel II.3. 


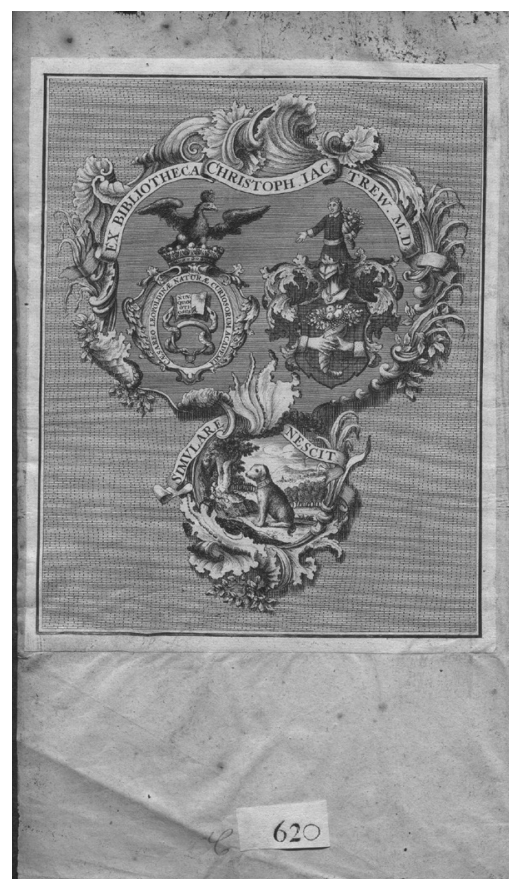

Trews dreiteiliges Exlibris (UBE H6l/2 TREW.C 620)

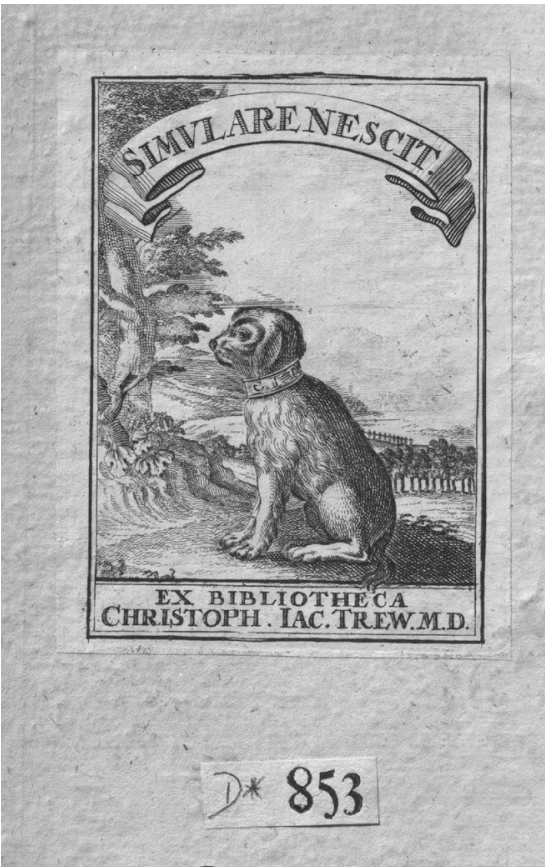

Trews erstes Exlibris mit Hund (UBE H61/TREW.Dx 853)

Das Wappenschild zeigt ebenfalls ein Füllhorn, gehalten von zwei vereinten Händen in der Figur der „Treuhand”: Die Linkshand zeigt dem Betrachter den Handrücken, die Rechtshand Daumen und Handballen, zwischen ihnen das Füllhorn. Diese „treuen Hände” symbolisieren die Kardinaltugend der Treue bzw. die Pflicht zur Wahrheit, das Füllhorn Fruchtbarkeit und Reichtum. Das zweite Motiv zeigt einen Hund. Ein kleiner Mischling sitzt in einer offenen Landschaft wartend vor einem Baum; sein Halsband trägt die Initialen seines Herrn C. I. T. Auf dem Spruchband darüber ist das Motto „Simulare nescit” - er kann sich nicht verstellen - zu lesen. Es steht vordergründig für die sprichwörtliche Treue des Hundes, mit den Initialen aber auch für Trew selbst. Der 
Wahlspruch, aus dem Namen heraus gewachsen, repräsentiert eine ethische Norm im Sinne des Bourdieuschen Habituskonzepts zur (Selbst-)Wahrnehmung und Beurteilung gesellschaftlicher Handlungen. Bereits um 1720 am Beginn seiner Berufslaufbahn, als sich Trew nach Studium und Gelehrtenreise als Stadtarzt in Nürnberg niedergelassen hatte, war das Hundemotiv für ein kleines Exlibris entstanden. Mit dem Motto verpflichtet sich der Gelehrte zur Wahrheit in der Wissenschaft, es enthält darüber hinaus und passend zur Exlibris-Funktion eine der wichtigsten Zuschreibungen an das Buch als Trägermedium kodifizierten, „wahren” Wissens. Als drittes Motiv für das große Exlibris nutzt Trew das Wappen der Leopoldina-Gesellschaft, in die er 1727 aufgenommen worden war. Es bestätigt seine anerkannte Position als Mitglied einer der bedeutendsten naturforschenden Gesellschaften seiner Zeit.

Ebenfalls am Beginn seiner Laufbahn und seiner Sammeltätigkeit legte Trew 1724 das erste von insgesamt fünf Stammbüchern an, in die sich die Besucher seiner Bibliothek, interessierte Reisende, Gelehrte und Freunde, einschreiben konnten. Trew führte diese Praxis bis unmittelbar vor seinen Tod 1769 weiter. Für die Stammbuchblätter hatte er einen

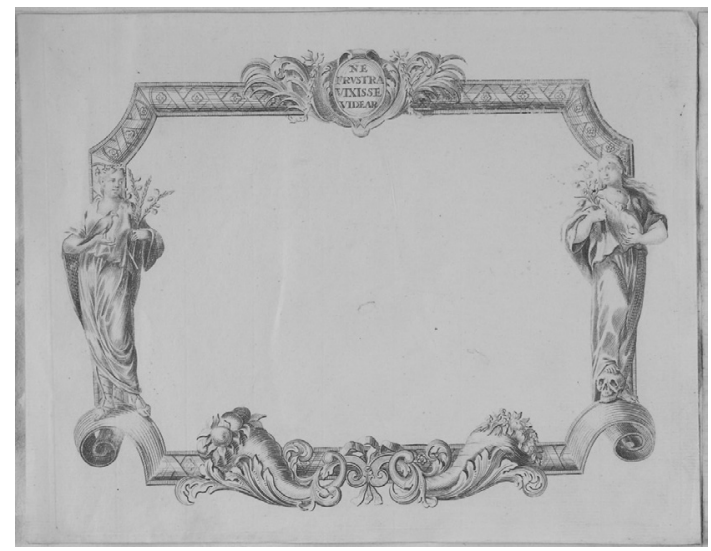

Stammbuchblatt mit Schmuckrahmen ohne Eintrag (UBE Stammbuch I, MS 1471) 
Schmuckrahmen in Auftrag gegeben, der von einer Kupferplatte im Rotdruck auf Blätter im Querfolio-Format übertragen und die nach der Beschriftung in Alben lose eingelegt wurden. Die Symbolik des Rahmens ist sorgfältig geplant. Er zeigt Motive, die auch in die verschiedenen Exlibris-Varianten aufgenommen wurden. In die untere Leiste sind in Anlehnung an das Familienwappen zwei mittig angeordnete Füllhörner eingearbeitet. Die Figur in der rechten Leiste, die Frühlingsgöttin Flora, trägt einen kleinen Hund auf der Handfläche, rechts ist Ceres zu sehen, die Göttin des Ackerbaus, der Ernte und der Fruchtbarkeit. Als Motto für die Kartusche der oberen Leiste hat Trew die angeblich letzten Worte des Astronomen Tycho Brahe von 1601 gewählt: „Ne frustra vixisse videar": "Lass mein Leben nicht vergeblich gewesen sein".51

Porträt, Exlibris und Stammbuch sind Dinge, die in hoch konventionalisierte Praktiken um Buch und Bibliothek eingebunden sind. Dass Trew zumindest Stammbuch und Exlibris schon sehr früh, und noch bevor er sich als erfolgreicher Stadtarzt, bekannter Sammler und Gelehrter profiliert hatte, in sein Dispositionssystem einbindet, zeugt von seinen bereits am Beginn seiner Karriere zutage tretenden Ambitionen.

Trews strategisches Handeln in sozialen Feldern

Die folgenden Bemerkungen gehen auf die Strategien ein, die Trew im ökonomischen, sozialen und kulturellen Feld übt und Teil des Habituskonzepts sind. Die unterschiedlichen Kapitalsorten setzt Trew von seiner Nürnberger Etablierung bis zu seinem Tod seinen Ambitionen entsprechend ein.

Trew war von Haus aus nicht vermögend, erst seine Tätigkeit als Stadtarzt und seine Heirat 1723 mit der reichen, älteren Witwe Magdalena Apollonia Trew, einer entfernten Verwandten, erlaubte ihm den hohen Einsatz von finanziellem Kapital auf dem kulturellen Feld, insbesondere zum Aufbau der Bibliothek, zur Anfertigung botanischer Zeichnungen sixteenth century. Cambridge 2014 [Reprint Edinburgh 1890], S. 309. 
und für seine Publikationen. Die Heirat mit Apollonia entspricht dem pragmatischen Konzept der frühneuzeitlichen Vernunftehe zum beiderseitigen Wohlergehen: die verwitwete Appollonia fand einen neuen, standesgemäßen Partner innerhalb der erweiterten Familie und war versorgt, der junge Arzt gewann neben Vermögen und dem damit verbundenen Status eine gute Haushälterin. Aus dieser, wohl glücklich passenden Verpaarung, gingen zu Trews Bedauern keine Kinder hervor.

Neben dem beträchtlichen Vermögen, das Appollonia in die Verbindung einbrachte, wuchsen Trews Geldmittel aus seiner Berufstätigkeit mit der Zeit beträchtlich an: bereits 1734 verdiente er als Stadtarzt in Nürnberg 800-900 Gulden pro Jahr, das Vierfache des Einkommens eines Württemberger Stadtarztes, und ab 1736 bezog er weitere 400 Gulden jährlich als Leibarzt der Markgrafen von Ansbach, ${ }^{52}$ eine Berufung die neben Geld auch eine Steigerung des Ansehens bedeutete.

Dass Trew als sehr vermögend galt, bezeugt auch folgender Brief von Johann Gesner 1747:

„Die Sammlung Ihro Exell[enz] Herrn Hofrath Trew übersteigt das Vermögen viler privatorum und ist etwas recht fürstliches; zu meiner Zeit (u[nd] 1728) waren selbst die Sammlung der gemahlten Pflanzen in der königlichen Bibliothec zu Paris noch nicht auf dise anzahl gekommen."53

Die Sammlung der botanischen Zeichnungen nennt Gesner "fürstlich". Trews Umgang mit Geld folgte, im Gegensatz zum „verschwenderischen” Adel, allerdings der bürgerlichen Tugend der Sparsamkeit. Die Praxis des guten Haushaltens führt Trew als Maxime in einem posthum, ein Jahr nach seinem Tod publizierten, Rückblick auf seine Sammeltätigkeit aus:

„Meine Sammlung, die ich seit 50 Jahren, zwar anfangs mit geringen Kräften, sparsam, nachmals aber durch göttlichen Segen reichlicher, mit allem Fleiß und Sorgfalt bey allen Gelegenheiten zusammen gebracht und

52 Vgl. EngL: Die medizinisch-naturkundliche Bibliothek des Nürnberger Arztes Christoph Jacob Trew, Kapitel II.l.

53 Brief von Johann Gesner an Christoph Jacob Trew vom 26.8.1747, Briefnr. 36, S. lv. 
die Kosten dazu an Gesellschaften, Kleidern, Bedienung und genauem Haushalten zu einem künftigen gemeinsamen Nutzen erspahret habe $[\ldots] . .54$

Die enge Verzahnung von ökonomischem, kulturellem und sozialem Kapital wird hier deutlich: die Sparsamkeit an Geld durch Verzicht auf Kleiderluxus, auf kostspielige Geselligkeiten und persönliche Annehmlichkeiten durch (überflüssiges) Personal. Als intellektuelles Kapital, das zum ökonomischen Vorteil eingesetzt wird, benennt er Fleiß und Sorgfalt, mit denen er bei „allen Gelegenheiten” Sammelobjekte erworben habe. Die Ökonomie des Wissens, Trews genaue Bücherkenntnisse, die Erfahrungen mit dem Buchmarkt und Auktionswesen, die Schätzung der angemessenen Preise und der günstigen Beschaffungswege sowie der Nutzung seiner Netzwerke in der „res publica litteraria” sind Geldeswert, obwohl Trew das Gelingen nicht allein eigenem Bemühen, sondern im protestantischen Wertekanon dem "göttlichen Segen” zuschreibt. Der Einsatz dieser Kapitalsorten geschieht zum zukünftigen "gemeinsamen Nutzen”. Ökonomisches und kulturelles Kapital schlagen in soziales und symbolisches um: gesellschaftliches Ansehen, Wertschätzung und Nachruhm.

Trew unterhielt einen umfangreichen und zeitraubenden Briefwechsel, eine unerlässliche Aktivität zur Pflege der gelehrten und freundschaftlichen Netzwerke. Diese waren Trägermedien eines ständigen Zirkulierens von Informationen, Gefälligkeiten und sonstigen Tauschgeschäften. Trews wissenschaftliche Projekte und der Aufbau seiner Sammlungen sowie seine Publikationen sind kaum ohne die Zuarbeiten seiner Kommunikationspartner und die vielfältigen Tauschbeziehungen, die in den Briefen aufscheinen, denkbar.

Dazu nur ein Beispiel. Der junge Naturforscher Trew beschäftigte sich mit der Großen amerikanischen Aloe (Agave americana L.), einer zu Nürnberg 1770, S. $17 \mathrm{f}$. 
dieser Zeit viel beachteten, exotischen Pflanze. ${ }^{55}$ Das Interesse wurde noch geschürt durch die Flugblätter und Kleinschriften, in denen die seltenen Blühereignisse festgehalten wurden. Trew plante, eine botanisch exakte Beschreibung der Arten, eine „Aloegraphia”, auf dem damaligen Stand der Klassifizierungsmethoden zu publizieren. Dazu war er auf die Mitarbeit von Freunden und Kollegen angewiesen, die ihm möglichst genaue Beobachtungen zum Habitus der Pflanzen und zu den Blühereignissen mitteilen sollten. Der folgende Brief zeigt anschaulich das Stakkato der Trewschen Fragen, Bitten und Anweisungen. Am 27. November 1731 schrieb er an den zu dieser Zeit in Regensburg wohnenden Freund und Apotheker Johann Ambrosius Beurer:

„ich habe darinnen [in meinem letzten Brief] gebetten die güte zu haben, und mir gelegentlich ein folium aloes Juccae folio in natura zu senden, gegenwärtig ersuche von folgenden eine beliebige Nachricht einzuziehen. 1) Wie alt die aloe beyläufig geschäzet wird. 2) in welchem Monat der stengel angefangen sich zu zeigen. 3) wie hoch der stengel von Blättern an u[nd] der Stamm vom erdreich biß zu anfang deß stengels geweßen. 4) $\mathrm{Ob}$ sich die Blumen bey nachtszeit mehr ausgebreitet oder zusammen gezogen. 5) ihre anzahl 6) ob gar kein saamen capsel angesezet. 7) wie der $\mathrm{H}[\mathrm{err}]$ Besizer deß Gartens heißet. 8) Ob die Blumen zu keiner Zeit einen merklichen Geruch verspühren laßen."56 Selbstverständlich war Trew auch bereit, die Bitten der Kollegen im Gegenzug entsprechend zu erfüllen.

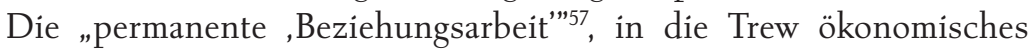
und kulturelles Kapital investiert, zeigt ihn als Akteur im Knotenpunkt von Kommunikationsnetzwerken. Die Beziehungsarbeit zahlt sich

55 D. Christophori Jacobi Trew, Republicae Norimbergensis Physici Ordinarii \& Academiae Caesareae Naturae Curiosorum Collegae Beschreibung Der Grossen Americanischen Aloe ... Wobey das tägliche Wachsthum des Stengels der in 1726. Jahr zu Nürnberg verblüheten Aloe in Tabellen mit Oberservationibus Metereologici erläutert, und die natürliche Beschaffenheit des reiffen Saaamens vorgestelltet wird. Nürnberg: Wolfgang Moritz Endter Erben und Julius Arnold Engelbrecht, 1727.

56 Brief von Johann Ambrosius Beurer an Christoph Jacob Trew vom 5.3.1732, Briefnr. 31.

57 Schwingel: Pierre Bourdieu zur Einführung, S. 92. 
als soziales Kapital aus. 1747 schrieb Trew an Josef von Rathgeb: „so kann ich ferner nicht unterlassen, Euer xx Nachricht zu geben, daß ich selbsten nach meinem Vermögen eine Bibiothecam medicam und was insbesondere auch zu historiam naturalum gehöret, $\mathrm{zu}$ sammlen (gesonnen bin): dann weil mir Gott kein Kind gegeben, so bestehet mein einziges Vergnügen darinnen und habe ich dabey die Absicht, wenn es Göttliche Schickungen nicht verhindern, dadurch mein Andenken bey der Nachwelt zu erhalten." 58

Letztendlich führt der strategische Einsatz von Kapitalien auf den gelehrten Praxisfeldern dazu, dass Trew sein selbst gesetztes Lebensziel erreicht. Das akkumulierte Sozialkapital ist in bedeutendes symbolisches Kapital eingegangen. Das Motto des Stammbuchs „Lass mein Leben nicht vergeblich gewesen sein", mit dem Trew sich früh in die Traditionslinie berühmter Gelehrtenleben eingereiht hatte, hat sich im Nachruhm erfüllt. Dies wird auch von der Umwelt bestätigt, wie u.a. ein Brief des Präsidenten der Leopoldina, Andreas Elias Büchner, 1752 an Trew anlässlich der Bitte um ein Büchergeschenk für die Erfurter Bibliothek der Akademie bezeugt:

„Ew. Wohlgeboren werden also daraus [s.c. aus einer dem Brief beigelegten Zeichnung der Erfurter Bibliothek] zu ersehen belieben, daß derjenige Zuwachs an Büchern, welche Dieselben vor diese unsere Bibliothek aus besonderer Generosité zu destiniren belieben, recht wohl aufbehalten wird, mithin also Dero unvergeßliches rühmliches Andenken desto besser auf unsere Nachkommen kann fortgepflanzet werden." 59

58 Brief von Christoph Jacob Trew an Joseph von Rathgeb von 1747, Briefnr. 639; hier zitiert nach Sснмidt-Herrling: Die Bibliothek des Arztes Christoph Jacob Trew, S. 92.

59 Brief von Andreas Elias Büchner an Christoph Jacob Trew vom 9.3.1737, Briefnr. 43; hier zitiert nach Sснмidt-Herruing: Die Bibliothek des Arztes Christoph Jacob Trew, S. 117. 
LES BIBLIOTHÈQUES ET L'ÉCONOMIE DES CONNAISSANCES Bibliotheken und die ÖKonomie des Wissens

$$
145^{\circ}-185^{\circ}
$$

Colloque international - Internationale Tagung 9-13 avril/April 2019 Sárospatak (Hongrie/Ungarn)

Édité par

Frédéric Barbier, István Monok et Andrea Seidler 


\section{L'Europe en réseaux}

Contribution à l'histoire de la culture écrite 1650-1918

Vernetztes Europa

Beiträge zur Kulturgeschichte des Buchwesens 1650-1918

Édité par

Frédéric Barbier, Marie-Elisabeth Ducreux, Matthias Middell, István Monok, Éva Ringh, Martin Svatoš

Volume VIII

École pratique des hautes études, Paris

École des hautes études en sciences sociales, Paris

Centre des hautes études, Leipzig

Bibliothèque nationale Széchényi, Budapest

Bibliothèque et centre d'information de l'Académie hongroise des sciences, Budapest 
LeS BIBLIOTHÈQUeS ET L'ÉCONOMIE DES CONNAISSANCES BibliotheKen und die ÖKonomie des Wissens

$$
145^{\circ}-185^{\circ}
$$

Colloque international - Internationale Tagung 9-13 avril/April 2019 Sárospatak (Hongrie/Ungarn)

Édité par

Frédéric Barbier, István Monok et Andrea Seidler

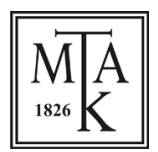

Magyar Tudományos Akadémia Könyvtár és Információs Központ Budapest 2020 
Mise en page

Viktória Vas

ISBN 978-963-7451-57-7

DOI 10.36820/SAROSPATAK.2020 
Préface

István MoNOK

Bibliothecae mutantur - Quare, quemadmodum et quid attinet?

Transformations de la composition thématique

des bibliothèques du Royaume de Hongrie aux $\mathrm{XV}^{\mathrm{E}}-\mathrm{XVI} \mathrm{I}^{\mathrm{e}}$ siècles....11

Marianne CARbonnier-Burkard

Les bibliothèques des Églises réformées françaises au XVII siècle.... 30

Max Engammare

De la bibliothèque de l'Académie de Calvin (1570) a la bibliothèque de l'Académie de Bèze (1612) à travers leur catalogue: Continuités et ruptures jusqu'au troisième catalogue de 1620........... 57

Róbert OLÁ́

Obsolescent Reformed Libraries in the seventeenth and eighteenth Century Carpathian Basin

Ádám Hegri

Moderner Zeitgeist - veraltete Lesestoffe. Bibliotheken reformierten Pfarrer um die Wende des 18. zum

19. Jahrhunderts im Königreich Ungarn

Petr MAšEK

Zierotin Library in Velké Losiny in Sixteenth and Seventeenth century. 136

Detlef HABERLAND

Schlesische Bibliotheken Zeichen der intellektuellen Vielfalt einer zentralen Bildungsregion in Europa 146

Thomas WaLLNIG

Sebastian Tengnagel und Johann Seyfried - Österreichische

Geschichtsschreibung zwischen Späthumanismus und

Gegenreformation 
Elisabeth EngL-Ursula RautenBerg

Christoph Jacob Trew - Bibliothek und Sammeln in der Gelehrtengemeinschaft der ersten Hälfte des 18. Jahrhunderts.

Helwi BLOM

Philosophie ou Commerce?

L'évolution des systèmes de classement bibliographique dans les catalogues de bibliothèques privées publies en France au XVIII ${ }^{\mathrm{e}}$ siècle.

Maria Luisa López-VIDrIero Abelló

Les meubles de la connaissance: façons de devenir sage à prix fixe.

Frédéric BARbier

Distinction, récréation, identité: la trajectoire des « romans» en France sous d'Ancien Régime.

\section{Andrea SeIdler}

Die praktische Bedeutung ungarischer Sammlungen und Bibliotheken für führende Gelehrte des Königreichs Ungarn im späten 18. Jahrhundert am Beispiel des Jesuiten Georg Pray (1723-1801).

\section{Olga Granasztói}

Se divertir: les enseignements de la bibliothèque d'une femme aristocrate hongroise à la fin du XVIII siècle

Christophe Didier

La naissance du théâtre „des boulevards”, ou Comment la banlieue entre en bibliothèque (1780-1830).

\section{Andrea De Pasquale}

La nascita delle riserve di libri antichi in Italia

Index des noms de personne et de lieu..... 